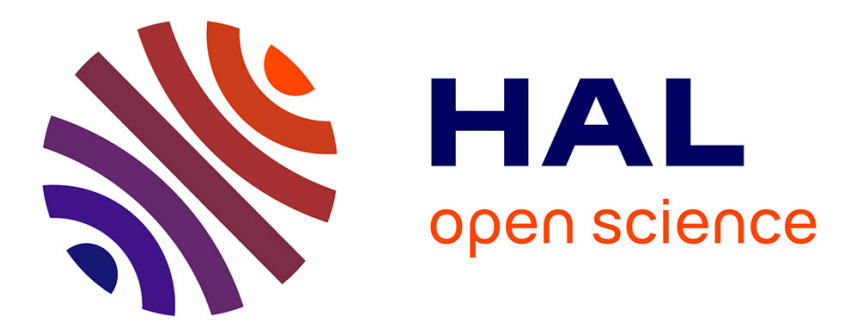

\title{
Effect of the velocities of the perturbers on alkali-rare gas line profiles
}

\author{
Nicole F. Allard, Y.G. Biraud
}

\section{To cite this version:}

Nicole F. Allard, Y.G. Biraud. Effect of the velocities of the perturbers on alkali-rare gas line profiles. Journal de Physique, 1982, 43 (3), pp.501-507. 10.1051/jphys:01982004303050100 . jpa-00209419

\section{HAL Id: jpa-00209419 https://hal.science/jpa-00209419}

Submitted on 1 Jan 1982

HAL is a multi-disciplinary open access archive for the deposit and dissemination of scientific research documents, whether they are published or not. The documents may come from teaching and research institutions in France or abroad, or from public or private research centers.
L'archive ouverte pluridisciplinaire HAL, est destinée au dépôt et à la diffusion de documents scientifiques de niveau recherche, publiés ou non, émanant des établissements d'enseignement et de recherche français ou étrangers, des laboratoires publics ou privés. 


\title{
Effect of the velocities of the perturbers on alkali-rare gas line profiles
}

\author{
N. F. Allard $(*)$ \\ Département d'Astrophysique Fondamentale \\ and Y. G. Biraud (**) \\ Astronomie Infra-Rouge, Observatoire de Paris, 92190 Meudon, France
}

(Reçu le 10 juillet 1981, révisé le 2 novembre, accepté le 26 novembre 1981)

\begin{abstract}
Résumé. - Nous étudions les effets de la température sur les profils de raie du Cs perturbé par du Xe dans l'approximation d'un potentiel carré. La simplicité de ce potentiel nous a permis pour la première fois de réaliser une moyenne sur les vitesses dans la théorie unifiée d'Anderson et Talman. Les modifications importantes portent sur les caractéristiques du satellite tant en position, largeur qu'asymétrie.
\end{abstract}

\begin{abstract}
We study the effects of temperature on line profiles of Cs perturbed by Xe in a square-well potential approximation. Its simplicity has allowed for the first time to perform an average on velocities in the unified theory of Anderson and Talman. The more important changes concern the satellite characteristics both for position, width and asymmetry.
\end{abstract}

1. Introduction. - The present contribution is the continuation of a previous study (Allard [1] and Allard and Biraud [2]) devoted to calculations of line profiles in the Anderson and Talman theory using a square-well interaction potential. This approach has proved to be a powerful aid in interpreting complicated experimental results because it can isolate the impact of each parameter thus providing good physical explanations of the problem. It is remarkable that this simple square-well potential, though very crude, yields computed spectral line profiles that are in satisfactory agreement with experimental data.

Because the line shape is obtained as the Fourier transform of an autocorrelation function long calculations have to be done. This is the main reason why calculations are restricted to the case of monocinetic perturbers except for those made in the oneperturber approximation [3].

In the Anderson and Talman theory a first approach has been made by Allard, Sahal-Bréchot and Biraud [4] who used a Lennard-Jones potential.

(*) C.N.R.S. (G.R. 24) Processus Atomiques et Moléculaires de l'Astrophysique, Groupe de Recherche au C.N.R.S.

$(* *)$ C.N.R.S. (L.A. 325), Astronomie Infra-Rouge, Laboratoire Associé au C.N.R.S.
But the complexity of the calculations imposed a rather crude integration which was not precise.

In the present situation with a square-well potential, the whole calculation of the autocorrelation is possible because its expression is semi-analytical (a few numerical integrations are nevertheless unavoidable), this is one of the advantages of the squarewell potential which allows to calculate a great number of profiles under various physical situations.

First we deal with the temperature effect on the profile when the perturbers are assumed to be monocinetic and then we will discuss the average over velocities and present our results.

2. Theory. - Line profiles are calculated using the theory of Anderson and Talman [5] and represent the Fourier transform (FT) of an autocorrelation function

$$
I(\Delta \omega)=\frac{1}{2 \pi} \int_{-\infty}^{+\infty} \Phi(s) \mathrm{e}^{i \Delta \omega s} \mathrm{~d} s
$$

where $\Phi(s)$ is calculated with the assumption that the radiators are stationary in space, the perturbers are mutually independent and the potentials are scalarly additive. $\Phi(s)$ is expressed by :

$$
\Phi(s)=\varphi(s)^{n}
$$


Table I. - Variation of the profile characteristics with $T$.

\begin{tabular}{|c|c|c|c|c|c|c|c|c|}
\hline \multirow[b]{2}{*}{$T(\mathrm{~K})$} & \multirow[b]{2}{*}{$v(\mathrm{~cm} / \mathrm{s})$} & \multicolumn{2}{|c|}{ LINE } & \multicolumn{5}{|c|}{ SATELLITE } \\
\hline & & Abs. Int. & $\begin{array}{r}\text { Width } \\
\left(\mathrm{cm}^{-1}\right)\end{array}$ & $\begin{array}{l}\text { Abs. Int. } \\
\times 10^{-2}\end{array}$ & $\begin{array}{c}\text { Rel. } \\
\text { Int./Line } \\
\times 10^{-2}\end{array}$ & $\begin{array}{r}\text { Width } \\
\left(\mathrm{cm}^{-1}\right)\end{array}$ & Position $\left(\mathrm{cm}^{-1}\right)$ & $\begin{array}{c}\text { Position in } \\
\text { cumulated } \\
\text { spectra }\left(\mathrm{cm}^{-1}\right)\end{array}$ \\
\hline 16 & $7 \times 10^{3}$ & 1.97 & 0.26 & 6.7 & 3.4 & 2.3 & -79.6 & -79.6 \\
\hline 63 & $1.4 \times 10^{4}$ & 1.06 & 0.49 & 3.36 & 3.2 & 4.6 & -79.5 & -79.6 \\
\hline 141 & $2.1 \times 10^{4}$ & $0.7^{\cdot}$ & 0.72 & 2.24 & 3.2 & 6.92 & -79.4 & -79.5 \\
\hline 251 & $2.8 \times 10^{4}$ & 0.53 & 0.94 & 1.68 & 3.2 & 9.24 & -79.3 & -79.5 \\
\hline 392 & $3.5 \times 10^{4}$ & 0.43 & 1.16 & 1.37 & 3.2 & 11.6 & -79.1 & -79.5 \\
\hline 565 & $4.2 \times 10^{4}$ & 0.34 & 1.48 & 1.14 & 3.3 & 13.9 & -78.9 & -79.4 \\
\hline 769 & $5 \times 10^{4}$ & 0.29 & 1.74 & 0.98 & 3.4 & 16.2 & -78.6 & -79.4 \\
\hline 1000 & $5.7 \times 10^{4}$ & 0.27 & 1.86 & 0.87 & 3.2 & 18.6 & -78.3 & -79.4 \\
\hline 1270 & $6.4 \times 10^{4}$ & 0.23 & 2.1 & 0.76 & 3.3 & 21 & -78 & -79.4 \\
\hline 1570 & $7 \times 10^{4}$ & 0.21 & 2.32 & 0.74 & 3.3 & 23.5 & -77.7 & -79.5 \\
\hline 1900 & $7.8 \times 10^{4}$ & 0.18 & 2.8 & 0.63 & 3.5 & 25.9 & -77.1 & -79.5 \\
\hline 2260 & $8.5 \times 10^{4}$ & 0.18 & 2.68 & 0.58 & 3.2 & 28.3 & -76.5 & -79.4 \\
\hline 2650 & $9.2 \times 10^{4}$ & 0.17 & 2.89 & 0.55 & 3.3 & 30.8 & -76.1 & -79.5 \\
\hline 3080 & $9.9 \times 10^{4}$ & 0.14 & 3.54 & 0.5 & 3.6 & 33.5 & -75.5 & -79.5 \\
\hline 3530 & $10^{5}$ & 0.13 & 3.94 & 0.49 & 3.7 & 36.1 & -75.1 & -79.5 \\
\hline
\end{tabular}

where $\varphi(s)$ is then the autocorrelation function in the binary hypothesis

$$
\begin{aligned}
\varphi(s)=\exp \left[-\int_{0}^{\infty} v f(v) \mathrm{d} v\right. & \int_{0}^{\infty} 2 \pi \rho \mathrm{d} \rho \times \\
& \left.\times \int_{-\infty}^{+\infty}(1-\exp i \eta(t, s)) \mathrm{d} t\right]
\end{aligned}
$$

$n$ is the perturber density, $f(v)$ the Maxwell velocity distribution, $\rho$ the impact parameter and $\eta(t, s)$ is the phase shift calculated along a classical path.

$$
\eta(t, s)=\int_{t}^{t+s} V\left(t^{\prime}\right) \mathrm{d} t^{\prime}
$$

the interaction potential is defined by :

$$
\begin{aligned}
V(R) & =V & & \text { if } & R \leqslant a \\
& =0 & & \text { if } & R>a
\end{aligned}
$$

where $R$ is the internuclear distance.
Then, the integrations over $t^{\prime}, t$ and $\rho$ are analytical. A comprehensive description has been done in [1] where the expressions of the autocorrelation function for monocinetic perturbers are given in equations (4) and (5). The last integration over $v$ is semi-analytical.

3. Monocinetic perturbers. - In our previous study [1], we have found periodic red satellites located at multiples of the depth of the well. The first satellite corresponds to the presence of one perturber in the interaction volume, the second to two, and so on. This has been discussed in [6] where our computed multiple satellites are corroborated by experimental results.

In the present study we have made calculations for a range of temperature varying from $16 \mathrm{~K}$ to $3530 \mathrm{~K}$. The main features of the line and of the 1st satellite are shown in table I.

3.1 STUDY OF THE LINE. - In figure $1 a$, we have plotted the variation of the width of the line against temperature. The curve oscillates around an increasing 


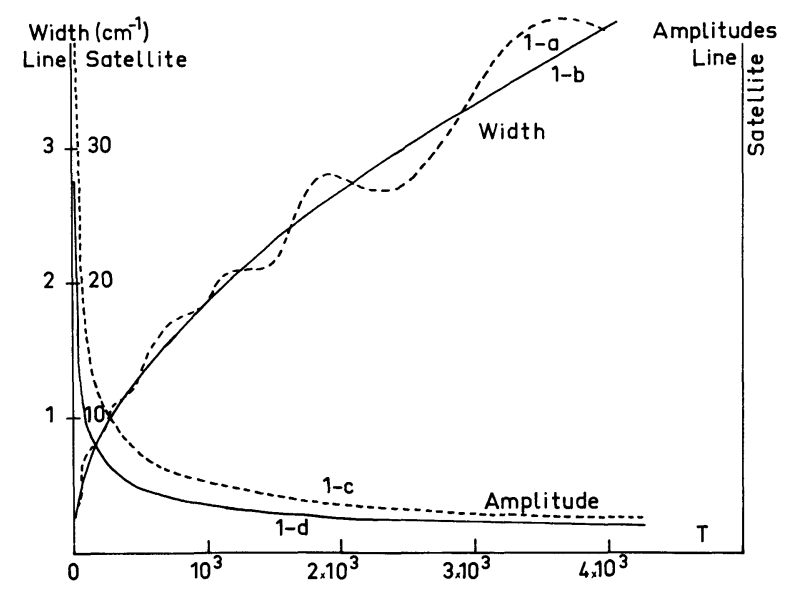

Fig. 1. - Variation with the temperature of : a) The width of the line ; b) The width of the satellite

c) The absolute amplitude of the line --------; d) The absolute amplitude of the satellite - .

parabolic curve (Fig. $1 b$ ) which represents the variation of width of the 1st satellite (see below). A physical interpretation of these oscillations seems difficult, they are perhaps due to the square-well shape of the potential.

In figure 2 we have plotted this variation with $\sqrt{T}$ and found it nearly linear. Due to the increasing of width, the intensity of the line decreases with temperature (Fig. 1c). The shift remains negligible, as the potential does not present a long range part, and as the satellite is well separated from the main line for the present potential and density.

3.2 Study of the Satellite. See table I. - The absolute amplitude of the satellite decreases in the same way as the line intensity (Fig. 1d). This is why the intensity of the satellite relative to the line remains constant.

As we have noticed, the width shows a parabolic variation but without oscillations. The satellite becomes closer and closer to the line as the temperature

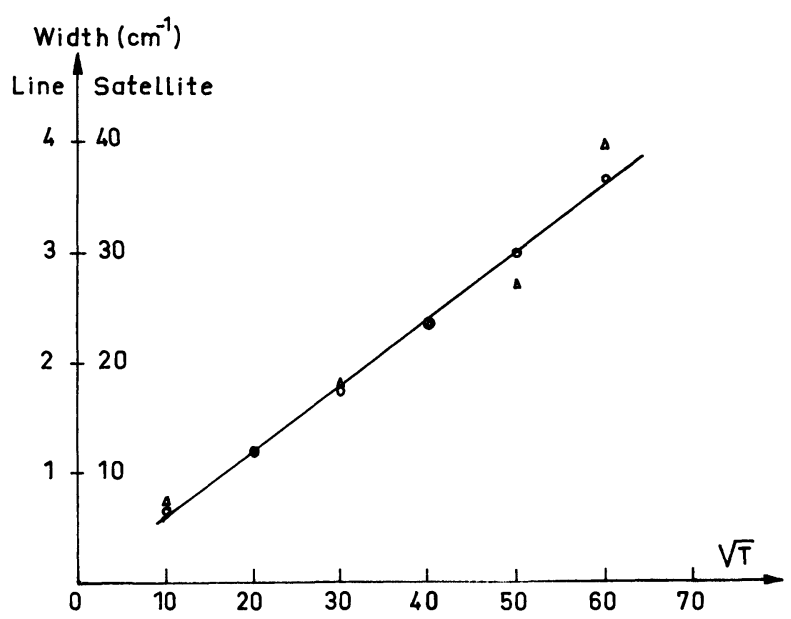

Fig. 2. - Variation with $\sqrt{T}$ of the width of the line : $\Delta$, and of the width of the satellite : $O$.

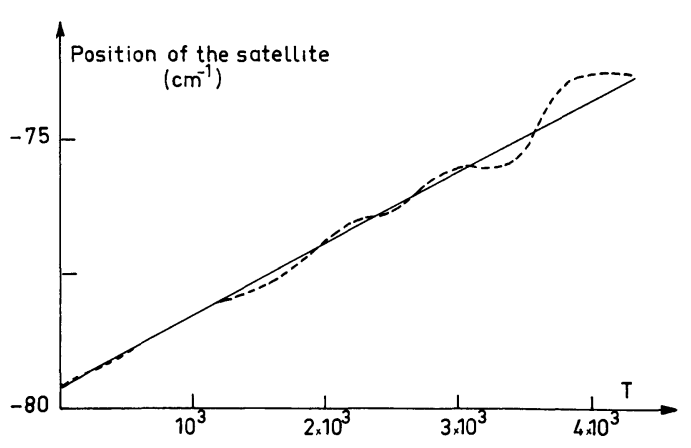

Fig. 3. - Variation of the position of the satellite with $T$. The curve is fitted by a straight line.

increases. The variation of the distance from the satellite to the line, with temperature, is linear for low temperatures (Fig. 3). The extrapolated value for $T=0$ gives the theoretical value predicted by the quasistatic approximation : $\omega_{\text {sat }}=-V$. Beyond $3000 \mathrm{~K}$, the oscillations appear again.

We have noticed that the temperature effect on the position of the satellite decreases with the interaction. A tentative interpretation is that the temperature broadening of both line and satellite makes them overlap inasmuch as they are less separated.

3.3 ConClusion. - For increasing temperatures :

- The widths of the main line and of the satellite increase.

- The distance satellite-line decreases regularly with $T$ until a given high limit temperature is reached. Then the line and satellite are no longer separated. This limit temperature evidently is smaller for weaker interactions.

- Line and satellite are symmetric when they do not overlap. So, temperature has no influence on the shape of the profile.

4. Average over velocities. - The complexity of.the computation of these profiles in a unified theory compels to assume monocinetic perturbers. An attempt to average with the Maxwell-Boltzmann velocity distribution has been done in [4] but the cost of the computation of one profile was too great to perform a detailed study. Only such a simple potential, allowing for an analytical expression for the autocorrelation function, gives a fast and precise computation of the velocity average, and permits to deal with a large variety of physical cases. Three approaches have been considered and they provide coherent results.

4.1 Methods of calculation. - 4.1.1 First method. - We have calculated the Maxwell-Boltzmann distribution $f(v)$ for $T=500 \mathrm{~K}$ and we have sampled it for 15 equispaced velocities $v_{i}$. Each $v_{i}$ is weighted by a normalized ponderation coefficient $\alpha_{i}$.

Then we can :

- Compute the corresponding $\Phi\left(v_{i}\right)$, average them, and Fourier transform $\left[\Phi\left(v_{i}\right)\right]_{\mathrm{Av}}$ for getting the profile $I(\omega)$ at $T=500 \mathrm{~K}$. 
- Fourier transform the various autocorrelations $\Phi\left(v_{i}\right)$ and average the $I_{v_{i}}(\omega)$.

The linearity of the FT leads to the same result. However, the 2nd method allows us for studying the intermediate cumulated spectra to compare step by step, the position of the 1 st satellite for each $v_{i}$ to the position obtained in the overall resulting profile (see table I).

4.1.2 Second method. - The direct calculation of the average uses a semi-analytical expression which provides a fast calculation of the profile.

The results obtained in the two methods are coherent but not identical, because in the first method the Maxwell-Boltzmann distribution is sampled and truncated for $v=10^{5} \mathrm{~cm} / \mathrm{s}$, while, in the second, the distribution is a continuous function.

\subsection{STUDY OF THE AUTOCORRELATION FUNCTION. -} For monocinetic perturbers the autocorrelation function presents oscillations only during the collision time $\tau_{\max }=2 a / \bar{v}$ and beyond it decreases exponentially as predicted by the impact theory (Fig. 2 in [1]).

Now, when we integrate on velocities, no such unique limit exists because the maximum collision time depends on $v: \tau_{\max }=2 a / v$. Hence the oscillations are no longer restricted to the finite domain $[0,2 a / \bar{v}]$ but will appear all along the autocorrelation function (Fig. 4).

Because the width of the satellite is of the order of the inverse of the collision time, one may see that the integration over $v$ which takes into account collision times greater than $2 a / \bar{v}$ will sharpen the satellite (Fig. 9).

In the monocinetic case the oscillations are artificially cut out beyond $2 a / \bar{v}$ which corresponds to the convolution of the satellite by a sinc function and gives rise to the so-called "diffraction oscillations" [1]. When integrating on $v$ this cut-off no longer exists and the amplitude of the oscillations falls down smoothly and the "diffraction oscillations " disappear (Figs. 6 and 7).

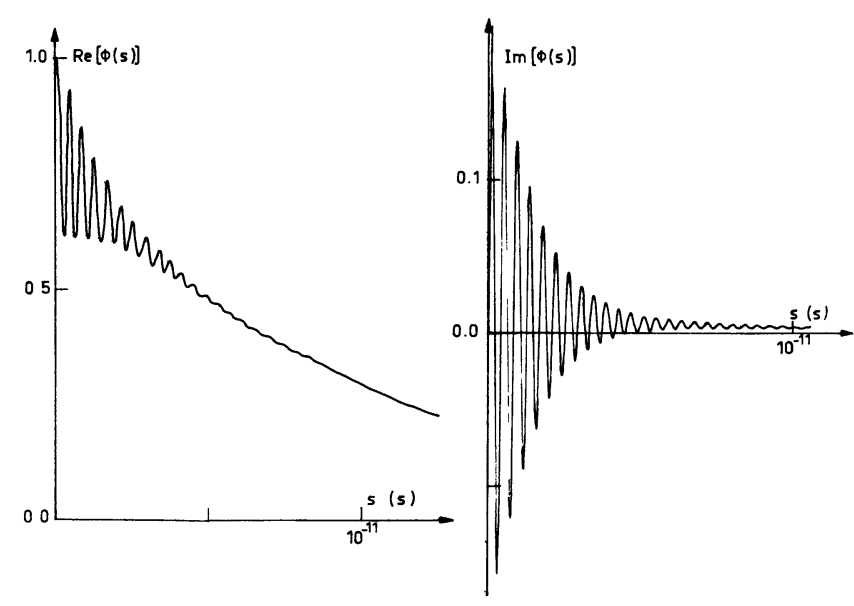

Fig. 4. - Real and imaginary part of the autocorrelation function.

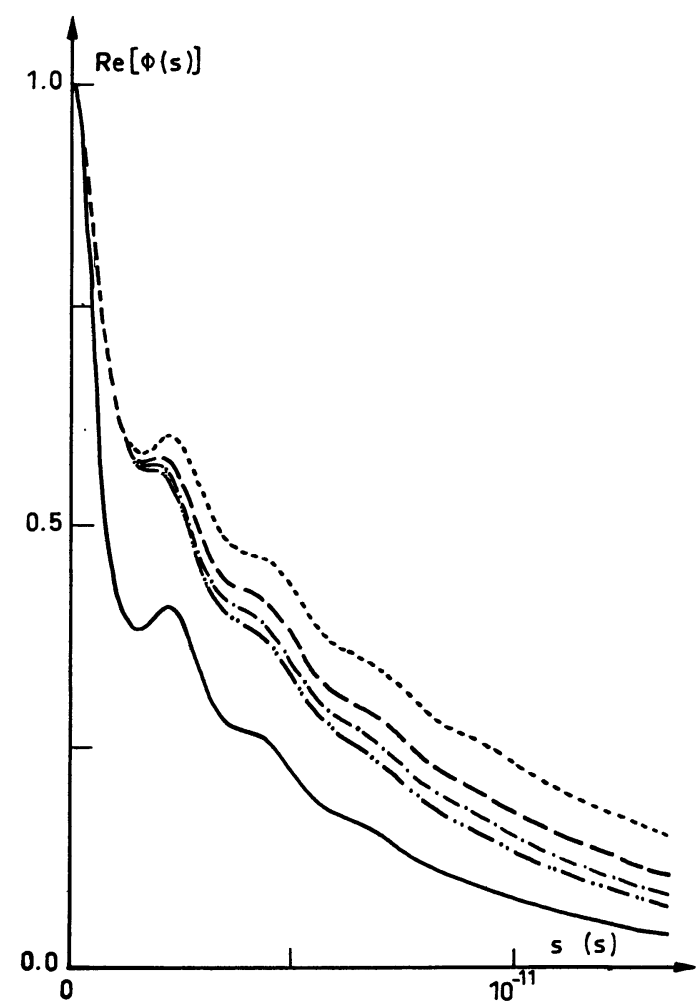

Fig. 5. - Real parts of the autocorrelation function for various $T:-T=400 \mathrm{~K}, h=0.65 ;-\cdots-.-T=400 \mathrm{~K}$, $h=0.25 ;--T=600 \mathrm{~K}, h=0.25 ;-\cdot T=700 \mathrm{~K}$, $h=0.25 ;-\cdots-T=800 \mathrm{~K}, h=0.25 . h=4 / 3 \pi a^{3} n$ ( $h$ is the number of perturbers in the interaction volume and $n$ the density).

We have plotted the autocorrelation function (Fig. 5) for a shallow potential, so the period of the oscillation is long. We can see that this period depends on neither temperature nor density. So $V$ remains the determining factor for the satellite-line separation. These results disagree with those obtained for monocinetic perturbers. But they are corroborated by the first method (study of cumulated spectra), where we notice that the evolutive position of the satellite

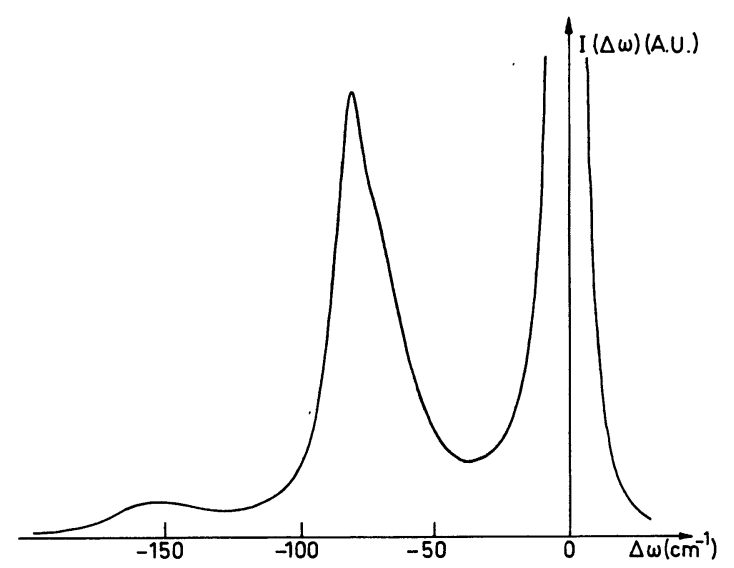

Fig. 6. - The profile for $V=-79.58 \mathrm{~cm}^{-1}$ and $T=3000 \mathrm{~K}$. 


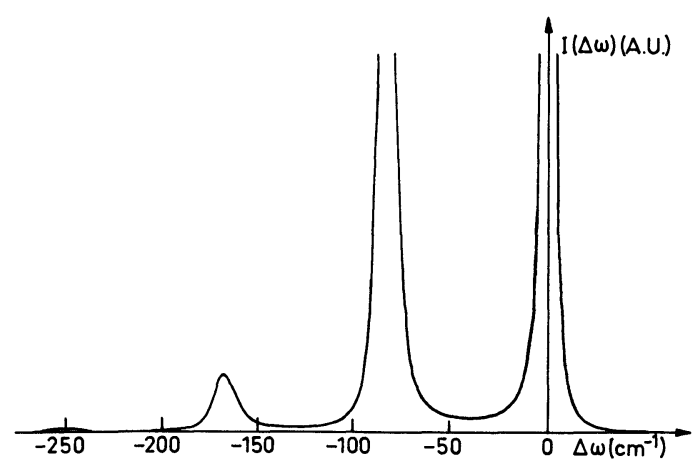

Fig. 7. - The profile for $V=-79.58 \mathrm{~cm}^{-1}$ and $T=300 \mathrm{~K}$.

remains the same as the quasistatic one which is preponderant because of the intensity and sharpness of the quasistatic satellite (Table I). This is one explanation but we have to remember that the shape of the Maxwell-Boltzmann distribution is also important. We notice also (Fig. 5) that the half-width of $\Phi(s)$ is larger for $400 \mathrm{~K}$ than for $600 \mathrm{~K}$. The line will be sharper for low temperatures than for high temperatures. When the temperature decreases, the velocity decreases, so the stay of the perturber inside the interaction volume is longer, the perturbed and nonperturbed wave trains are longer, which leads to sharper satellite and line.

5. Study of the main line (well resolved satellite). The potential chosen is $V=3 V_{\mathrm{T}}=-79.58 \mathrm{~cm}^{-1}$.

$V_{\mathrm{T}}$ is a remarkable value of $V$ defined in [2] and appeared to be the limit-value between the two situations : well-resolved and unresolved satellites.

We verify on table II that the variation of the width of the main line with $T$ is exactly as predicted by the study of the autocorrelation. The values of table II are the same for the monocinetic case.

Because the satellite remains well separated from the line and the density is small in the present study the shift is zero and the asymmetry $\left({ }^{1}\right)$ one [2].

( $\left.{ }^{1}\right)$ The asymmetry is defined as :

Blue Half-width Red Half-width
Fable II. - Variation of the width of the main line with $T$.

$\begin{array}{cccccc}T(\mathrm{~K}) & 300 & 400 & 500 & 600 & 700 \\ - & - & - & - & - & - \\ \text { Width }\left(\mathrm{cm}^{-1}\right) & 1.03 & 1.19 & 1.33 & 1.46 & 1.57\end{array}$

6. Study of the satellite $\left(V=-79.58 \mathrm{~cm}^{-1}\right)$. The temperature range is 300 to $3000 \mathrm{~K}$. On figures 6 and 7, we present the profiles corresponding to these two limit temperatures. We may notice that the first satellite becomes asymmetrical. The satellite for $300 \mathrm{~K}$ is sharp and well resolved: we tend to the quasistatic limit. On the contrary, at $3000 \mathrm{~K}$, their broadening leads to a rather important overlap.'

We have plotted the first satellites separately and present their evolution on figure 8. Here also all the properties deduced from the study of the autocorrelation functions are valid.

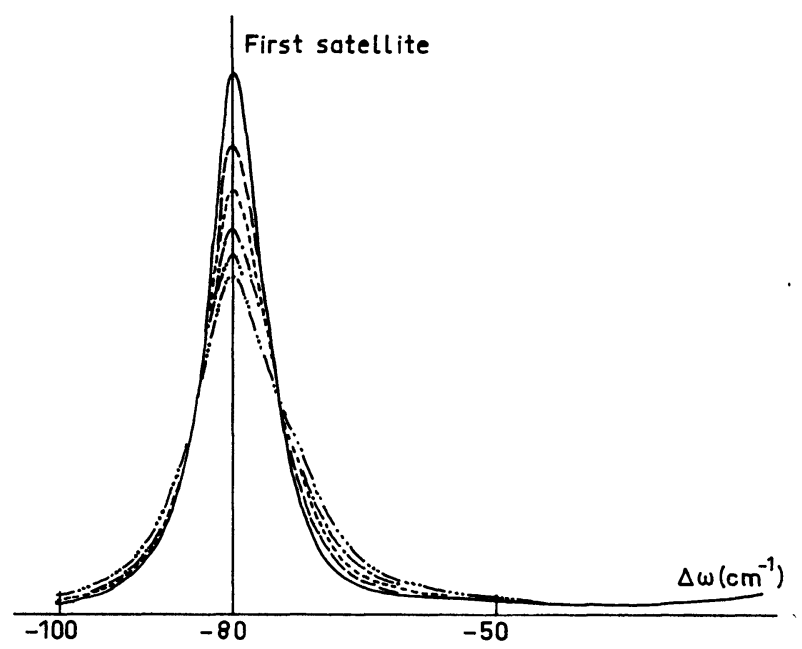

Fig. 8. - The variation of the first satellite with $T$ : $T=300 \mathrm{~K} ;--T=400 \mathrm{~K} ; \cdots T=500 \mathrm{~K}$; $-.-T=600 \mathrm{~K} ;-\cdots-T=700 \mathrm{~K} ;-\cdots-T=800 \mathrm{~K}$.

\subsection{Position OF THE FIRST SATELlite}

The examination of table III shows that the position of the satellite is the same whether the integration on $v$ is performed or not and is independent of $T$. This had been already predicted by the study of the autocorrelation function (Fig. 5) where one notices that the oscillation period does not depend qualitatively on $T$. If the position of the satellite were defined as the abscissa of its centre of gravity, we would have noticed a variation because of the asymmetry.

Table III. - Variation of the position of the satellite with $T$.

\begin{tabular}{llllll}
\multicolumn{1}{c}{$T(\mathrm{~K})$} & 300 & 400 & 500 & 600 & 700 \\
$\overline{-}$ & $-\overline{79.25}$ & $-\overline{79}$ & $-\overline{78.75}$ & $-\overline{78.75}$ & $-\overline{78.75}$ \\
Position $\left(\mathrm{cm}^{-1}\right)$ with $\bar{v}$ & -79.75 & -79.75 & -80 & -80 & -80 \\
With average on $v$ & &
\end{tabular}




\subsection{WIDTH OF THE SATELLITE.}

Table IV. - Variation of the width of the satellite with $T$.

$\begin{array}{ccccccc}T(\mathrm{~K}) & 300 & 400 & 500 & 600 & 700 & 800 \\ \begin{array}{c}\text { Width }\left(\mathrm{cm}^{-1}\right) \\ \text { with } \bar{v}\end{array} & 10 & 11.75 & 13 & 14.25 & 16 & 16.75 \\ \begin{array}{c}\text { With average } \\ \text { on } v\end{array} & 7.75 & 8.75 & 9.75 & 11 & 11.75 & 12.25\end{array}$

As explained before $(\S 4.2)$ the width of the satellite is less after integration on $v$ and increases with $T$.

\subsection{ASYMMETRY OF THE SATELlite.}

Table V. - Variation of the asymmetry of the satellite with $T$.

$$
\begin{array}{ccccccc}
T(\mathrm{~K}) & 300 & 400 & 500 & 600 & 700 & 800 \\
\overline{-} & - & - & - & - & - & - \\
\text { With } \bar{v} & 1 & 0.96 & 1 & 1.03 & 1.06 & 0.97 \\
\text { With average on } v & 1.21 & 1.33 & 1.44 & 1.44 & 1.42 & 1.45
\end{array}
$$

The satellite presents now an asymmetry. This is the new and interesting result obtained by the integration on $v$.

When the perturbers were supposed to be monocinetic, we found symmetrical satellites the position of which seemed to depend on the well depth only. In these conditions the mean of such satellites would never lead to an asymmetrical resulting satellite.

In paragraph 3.2 and 4.1 and in table I, we have presented the variation of the position of the satellite with $T$. This explains very well the asymmetry. Now the satellites, even though each of them is symmetrical, sum up into an asymmetric satellite whose maximum is very slightly shifted compared to the maximum of the monocinetic satellite corresponding to $500 \mathrm{~K}$. This can be explained by the important weighting of the small velocities by the Maxwell-Boltzmann distribution and mainly by the large magnitude of the satellite for low temperatures (see Fig. 9).

To our knowledge no experimental study of the effect of the variation of the temperature on the satel-

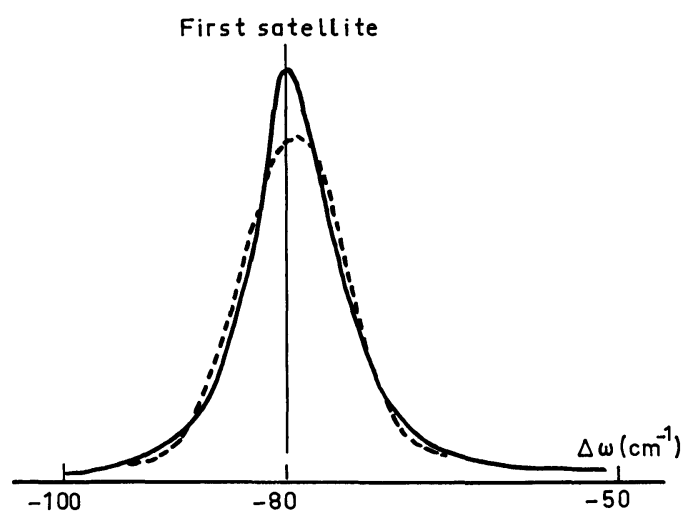

Fig. 9. - The first satellite :- With average on $v ; \ldots$ Without average. $T=400 \mathrm{~K}$. lite characteristics has been done except for partially resolved satellites [7] and [8]. In such a situation the blend does not allow a rigorous study comparable to ours.

7. Study of the main line (unresolved satellite). In this section we have fixed $V=\frac{V_{\mathrm{T}}}{2}=-13.27 \mathrm{~cm}^{-1}$. We have re-made all the calculations presented in [2].

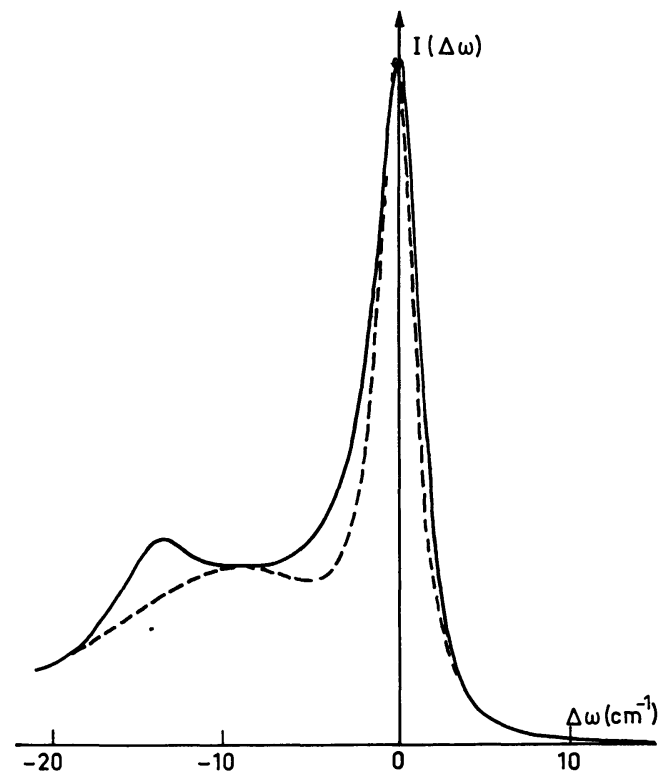

Fig. 10. - The profile for $V=-13.27 \mathrm{~cm}^{-1} ; T=400 \mathrm{~K}$; $h=0.65:-$ With average on $v ;----$ Without average.

On figure 10 , we compare the monocinetic profile to the average one. After integration on $v$, the satellite becomes asymmetrical and its centre of gravity is shifted towards the low frequencies. That is why,

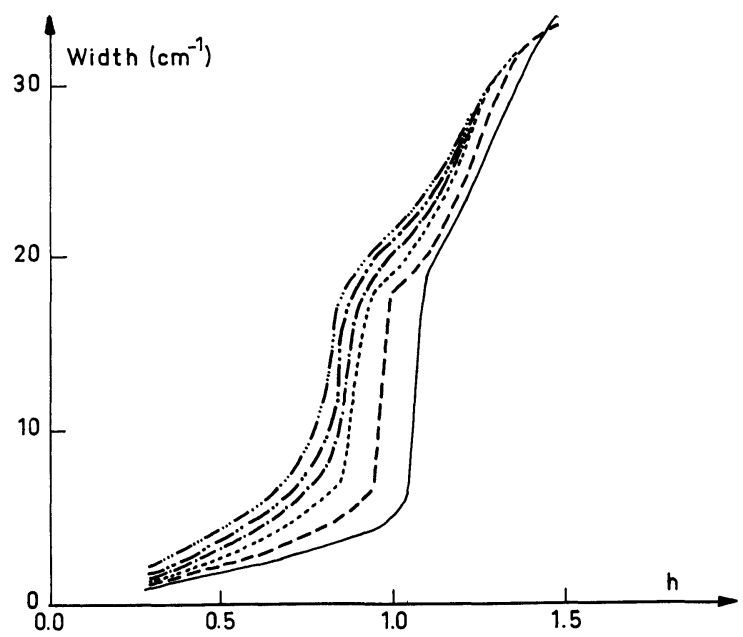

Fig. 11. - Variation of the width of the satellite with $h$ for various $T:$ With an average on $v:-T=400 \mathrm{~K}$; Without average : $-T=400 \mathrm{~K}, \cdots T=500 \mathrm{~K}$, $-.-T=600 \mathrm{~K},-\cdots-T=700 \mathrm{~K},-\cdots-T=800 \mathrm{~K}$. 
opposite to the results obtained with a well resolved satellite the position of which was invariant, we notice here a shift due to the important line-satellite overlap.

Nevertheless, its relative intensity is greater and, because it is sharper, it appears as better resolved. For the same reasons, on figure 11 the variations of $w$ with $h$ present analogous shape. But we may notice that the discontinuity appears for a smaller density after average on $v$. The same phenomenon is observed for the shift.

We have plotted on figure 11 a set of curves showing the variation of the line-width with density for a variety of temperatures : 400, 500, 700 and $800 \mathrm{~K}$. The evolution with $T$ is well explained by the increase of the overlap.
8. Conclusion. - This study was made possible by the use of a square-well potential. This is why it is new and has been never made in such details.

The integration on velocities has pointed out the following new phenomena for the satellite characteristics. Its width is smaller. Its position is no longer temperature dependent, while for monocinetic perturbers it was shifted towards the main line when the temperature increases. In compensation, a significant asymmetry appears.

We have not noticed any change about the line parameters but our square-well potential model was not fitted for its study. The addition of a long range part to the potential would be better for this purpose.

\section{References}

[1] Allard, N. F., J. Phys. B 11 (1978) 1383.

[2] Allard, N. F. and Biraud, Y. G., J. Quant. Spectros. Radiat. Transfer 23 (1980) 253.

[3] Sando, K. M. and Wormhoudt, J. C., Phys. Rev. A 7 (1973) 1889.

[4] Allard, N. F., Sahal-Brechot, S. and Biraud, Y. G., J. Phys. B 7 (1974) 2158.

[5] Anderson, P. W. and Talman, J. D., Proc. Conf. Broadening of Spectral Lines, Bell Telephone
System Technical Publications, № 3117, 29, Murray Hill, N.J. (1956).

[6] Kielkopf, J. F. and Allard, N. F., Phys. Rev. Lett. 43, 3 (1979) 196.

[7] Granier, R. et Granier, J., C.R. Hebd. Séan. Acad. Sci. Paris 262 (1966) 605.

[8] Ch'en, S. Y., Loot, E. C. and Garrett, R. O., Phys. Rev. 155 (1967) 38. 